\title{
The Boom Province: A Syndemic Approach to the HIV/AIDS Explosion among Aboriginal Persons in Urban Saskatchewan
}

\author{
Andrea Paige Cessna*
}

\begin{abstract}
The correlation between poverty and the increasing number of HIVIAIDS cases is striking in Saskatchewan's urban centres. Recent research indicates that the current spike of HIVIAIDS cases in the province disproportionately affects young Aboriginal women who live in Saskatoon's inner-city neighbourhoods. Because HIV/AIDS is both a pathological and social reality, this paper uses a syndemic approach to analyze coinfection, injection drug use, poverty, gender, and ethnicity/race as they pertain to the emerging HIVIAIDS epidemic in Saskatchewan. Taking into consideration Canada's colonial history and ongoing neo-colonialism facing Aboriginal persons, this paper explores the relationship between health configurations and larger political and economic systems that result in structural violence.
\end{abstract}

Keywords: medical anthroplogy, Aboriginal, Saskatchewan, syndemics

The correlation between poverty and contagious disease is disconcerting and has become increasingly evident in urban including medical anthropology. In 2010, the Saskatchewan Ministry of Health released an annual report providing an epidemiological review of human immunodeficiency virus (HIV) and acquired immunodeficiency syndrome (AIDS) surveillance data in the province. The findings in the report illustrated a steady increase in the annual number of HIV diagnoses in the last ten years, from 26 cases in 2002 to a peak of 200 cases in 2009 (Saskatchewan Ministry of Health 2011). The 2010 report also revealed a majority of Saskatchewan HIV cases in 2010 reported Aboriginal heritage with a significant percentage of these cases being settings within the realm of the health social sciences,

found in Saskatoon, Saskatchewan's largest city (Saskatchewan Ministry of Health 2011).

Biomedically, AIDS is not one disease. Rather, it is a composite of symptoms, conditions, and infections. Caused by HIV, AIDS is the result of viral damage to the immune system increasing the body's vulnerability to additional infections (Downe 2012). However, Hays challenges this biomedical stance saying, "disease is both pathological reality and social construction" [emphasis added] (2000:2). As such, the escalating number of Aboriginal cases of HIV in Saskatchewan is indicative of the need to go beyond solely biomedical explanations and investigate how social phenomena impact health. Syndemic theory provides a way to recognize the interconnections between health

*Department of Archaeology and Anthropology, College of Arts and Science, University of Saskatchewan, Saskatoon, SK, Canada Correspondence: apco56@mail.usask.ca 
configurations and larger political and economic systems that yield social inequality, structural violence, and inadequate public policies (Singer 1995:101). By taking into consideration social determinants of health and exposing "environments of risk and agents promoting risk, not just groups at risk and risky behavior," (Nichter 2008:157) attention can be focused upon the broader sociocultural factors fostering HIVIAIDS. This paper will argue Aboriginal people are subjected to an increased risk of contracting HIV/AIDS in urban Saskatchewan due to inequality and structural violence. Beyond providing an understanding of the terms 'syndemic' and 'risk environment,' this paper will use three main criteria to develop this argument: (1) a statistical illustration of the disproportionate number of HIV/AIDS cases among Aboriginal people; (2) a syndemic analysis of four factors (coinfection, injection drug use, poverty and social determinants of health) and their correlation to HIVIAIDS in urban Saskatchewan; and (3) how structural violence reinforces environments of risk, enabling the vicious cycle of poor health among Aboriginal peoples.

\section{Syndemics and Risk Environment}

The term "syndemic" refers to "a dynamic relationship involving two or more epidemic diseases or other disorders and the socioenvironmental context that promotes their interaction" (Singer 2009:29). The syndemic perspective flushes out the often-determinant sociocultural origins of disease while recognizing the fundamental importance of the biomedical construction of disease conception, and the disease-host interaction (Singer 2009:30). Put succinctly, when disease is looked at biomedically, it is often reduced to biochemical or physiological problems and treated biomedically; the syndemic approach rejects this narrow conceptualizing of disease and demands a broader perspective. Moreover, syndemics takes into consideration the 'social determinants of health,' referring to the notion that "health and illness are determined by life circumstances [and] that the most important factors that determine people's health are social" (Meili 2012:23).

A relevant example is the SAVA (Substance Abuse, Violence, and AIDS) syndemic, as Singer (2009) states:

From the syndemic perspective, AIDS, drug use, and violence in particular social contexts are so entwined with each other and each is so significantly shaped by the presence of the other two that if one tries to understand them as distinct things in the world it is hard to conceive of them accurately (31).
In this case, all three health components are interactive: drug use interacts directly with AIDS, worsening both conditions; similarly, violence and AIDS interact in mutually accelerating ways; and violence and drugs also propel one another (Singer 2009:31). These interactions are underlined by local social conditions and structural relationships tied to political and economic forces (Singer 2011:140).

"Risk environment" is defined as "the space-whether social or physical-in which a variety of factors, exogenous to the individual, interact to increase the chances of HIV transmission" (Rhodes et al 2005:1027). To assess the increased likelihood of Aboriginal persons contracting HIVIAIDS in Saskatchewan, it is paramount to consider the "social situations, structures and places in which risk is produced rather than a reliance on a conception of risk as endogenous to individuals' cognitive decision-making" (Rhodes et al 2005:1027).

\section{Urban Saskatchewan}

While the Canadian rate of HIV cases by year has remained relatively stable, Saskatchewan's crude rate of HIV surpassed that of the national average in 2006 and increased nearly 400 percent between 2004 and 2009 (Hammond et al 2011; Saskatchewan Ministry of Health 2011). The incidence of HIV in Saskatchewan is the highest in the country, approximately 2.5 times the national average (19.3 versus 7.2/100,000) (Adam 2011; Skinner 2011). Most relevant to the focus of this paper, the majority of HIV cases in Saskatchewan in 2009 were in urban centres, and 45 percent of these were located in the Saskatoon Health Region at the high rate of 31.3/100,000 (Saskatchewan Ministry of Health 2011). Nationally, the presence of HIVIAIDS has a remarkably harmful impact on Canada's Aboriginal community. A surveillance report by the Public Health Agency of Canada (2010) states, "25.4 percent of all HIV reports [in Canada] were among those identifying as Aboriginal." Furthermore, "Aboriginal people ... have a greater proportion of HIV among the age group of 15-19 years (4.9 percent)." In Saskatchewan, Aboriginal persons represent 73 percent of newly diagnosed HIV cases. Female cases who self-report Aboriginal ethnicity is at 86 percent while male cases is at 65 percent (Saskatchewan Ministry of Health 2011).

In his Sask HIV Update (2011), Stuart Skinner found that 50 percent of HIV positive patients admitted to hospitals in Saskatchewan progress to AIDS within three years of diagnosis. Skinner associates four main factors with this fast progression of HIV to AIDS in Saskatchewan: coinfections, such as tuberculosis and hepatitis $C_{\text {.; }}$ injection drug use (IDU); poverty, and other social determinants of health. By exploring these four factors, it is possible to expose how they are not only syndemic to HIV (wherein they exacerbate and accelerate the process to AIDS among 
Aboriginal people in urban Saskatchewan) but are also syndemically inseparable from one another.

\section{Coinfection}

Coinfection refers to two simultaneous infections interacting synergistically within the human body, such as HIV and hepatitis C (HIV/HCV) or HIV and tuberculosis (HIV and TB). In Introduction to Syndemics (2009:77), Singer refers to HIVIAIDS as an open door to numerous other infections and disorders. The study Health Disparity by Neighborhood Income, conducted by Lemstra, Neudorf, and Opondo (2006), indicated that the residents in six innercity, low-income neighbourhoods of Saskatoon experienced higher rates of poor health. In fact, these residents were nearly 15 times more likely to contract a sexually transmitted disease (STD) and 35 times more likely to contract hepatitis C (HCV) than Saskatoon's five most affluent neighbourhoods (Lemstra et al 2006:437). Both STDs and HCV are of concern for HIV positive individuals because TDs can cause lesions that enhance the ability of HIV to infect human bodies while "in Western countries, liver disease [caused by HCV] is the second leading cause of death among people with HIV/AIDS" (Singer, 2009:81). In his work A Healthy Society (2012), Ryan Meili identified the same inner-city, low-income neighbourhoods mentioned in the above Saskatoon study as being largely occupied by First Nations, Métis, and ethnic minorities. By virtue of living within impoverished neighbourhoods, Aboriginal people are at an increased likelihood of poorer health and, consequently, contracting HIV/AIDS.

In Saskatchewan tuberculosis is the 'opportunistic infection' in combination with HIV. Opportunistic infections are "diseases that generally are able to infect only individuals with compromised immune systems" (Singer 2009:225; Skinner 2011). The interaction between HIV and TB can take several forms and is bidirectional, meaning they affect each other reciprocally, as was seen of drugs/violence, AIDS/drugs and violence/AIDS within the SAVA syndemic. Further, malnutrition often accompanies these infections, creating a "triple threat" for human health due to mutually exacerbating conditions and accelerating the progression from HIV to AIDS. The interactions among HIV, TB, and malnutrition are problematic for Aboriginal people living in urban settings, such as Saskatoon's lowincome areas where there are significant deficits in services including safe housing, health services, and access to nutritious food. Health is impacted by social conditions and inequality which contribute to syndemical enhancement of disease (Claire, Singer 2003:429; Meili 2012:21). Moreover, Saskatchewan has one of the highest rates of tuberculosis among Aboriginal peoples compared to the average rate of Aboriginal populations in other provinces (44.3 versus 28.2/100,000) (Skinner, 2011). The correlations between
HIV and HCV, HIV and TB, and HIV and malnutrition illustrate these are "not just coinfection but enhanced infection due to disease interaction" (Singer \& Claire 2003:425), making it clear various social and physiological factors clash in the urban setting of Saskatoon's lowincome neighbourhoods. It is difficult to separate these three health components because each is simultaneously influenced by and influencing one another. Coinfection is a viable risk for Aboriginal people living with HIV/AIDS, yet it is heightened by IDU.

\section{Injection Drug Use}

Saskatchewan's HIV Strategy 2010-2014 (2011) states' "the pattern of HIV in Saskatchewan is different from any other province or territory in that the new cases are predominantly injection drug users (60\%) and/or Aboriginal (72\%)." Not unlike the substance abuse seen in the SAVA syndemic, IDU plays a major role in exacerbating already poor health by "promoting HIV transmission, disease progression, AIDS-related mortality and the development of AIDS defining illnesses" (Singer 2011:161). Nationally, Aboriginal persons have the highest proportion of HIV related to IDU at 60.3 percent (Public Health Agency of Canada 2009).

Imperative to assessing the high number of Aboriginal IDU-related cases of HIV in Saskatchewan is "recognizing the events and structures, both past and present, that have contributed to substance abuse, migration, unemployment, cultural displacement and despair among Aboriginal people in Canada" (De Bruyn 1998:19). By and large, these are results of colonization in Canada; in Saskatchewan, many Aboriginal persons use drugs and alcohol to cope with homelessness, discrimination, and violence or "to fit in and numb the overwhelming feelings of shame and guilt that [invade] every aspect of their lives" (Romanow 4.2.12).

There are various reasons why IDU is so prevalent among HIV positive persons and Aboriginal persons. In many instances, IDU is linked with the sexual transmission of HIV as a consequence of sex work in order to pay for addiction. Another factor contributing to IDU among Aboriginal persons is sharing needles and injection equipment. This is often the result within a contained social setting; for instance, family members may share equipment while socializing or to demonstrate 'loyalty' (Saskatchewan Ministry of Health 2010). Individuals with IDU-related HIV may progress to AIDS faster because abstaining from drug use is difficult, and, as a result, they may not attend treatment or may leave the hospital during their stay. Syndemically, this draws attention to "the vertical linkages that connect a social group or behavior to larger political and economic systems and to the arrangement of social relationships they help produce and reproduce over time" (Singer 2011:160). Among the many repercussions 
colonization has on Aboriginal peoples in Canada, an archetype sustaining negative social behaviors (i.e., drug use) and the intergenerational trauma of poor health is poverty.

\section{Poverty}

Across Canada, Aboriginal families are three times more likely to find themselves in poverty as non-Aboriginal families (Meili 2012:65). According to the study Health Disparity by Neighbourhood Income (Lemstra et al 2006:436), the average annual family income in Saskatoon's six inner-city neighbourhoods was approximately 30,000 dollars per year, while in the wealthiest neighbourhoods the average annual income was just under 100,000 dollars per year. The families in the wealthiest neighbourhoods were substantially healthier and significantly less likely to experience suicide attempts, contract STDs, or face violence (Lemstra et al 2006:437). Geographic research examining the marginalization of poor neighbourhoods in a Saskatoon study found that "supermarkets are not equally accessible in all urban neighbourhoods, with inner cities or areas with low-income populations having less access" (McCreary, Peters 2008:80). This leaves residents of lowincome neighbourhoods to access food at convenience stores where food is more expensive, high in calories, and poor in nutrition (McCreary, Peters 2008:80).

Among Aboriginal persons in Saskatchewan, poverty contributes to feelings of powerlessness, stemming from the effects of racism and colonization (Romanow 2003: 2.4). DeBruyn argues that in Canada, "people who were marginalized, stigmatized and discriminated against before HIV/AIDS arrived, have become over time those at highest risk of HIV infection" (1998:18). The HIV and AIDS in Saskatchewan 2010 report echoes this stating:

HIV/AIDS has the greatest impact on Canadian populations already vulnerable to a range of health, social and economic inequities. The social determinants of health that impact risks of acquiring HIV, especially injection drug use, include factors such as poverty, inadequate housing, lack of education or job training, child abuse and family violence (Saskatchewan Ministry of Health 2010).

Romanow adds, "poverty affects attitudes to conscious risktaking in complex ways" (2003:2.1). For example, an individual may decide not to use condoms due to cost or in spite of restricted access to STD treatment. In other cases, low-income Aboriginal women, whose negotiating position with sexual partners may be undermined by economic dependence, may not use adequate protection during sexual intercourse (Romanow 2003: 2.4). Often, individuals who live in poverty are forced to neglect their disease to cope with immediate risks in their lives, such as homelessness, food insecurity, and poor socio-economic supports. Finally, stress from discrimination, stigma, and social suffering is another common consequence of poverty (Singer \& Claire 2003:430). Thus, the interactions between poverty and health are numerous. Poverty plays a powerful role that affects all other social determinants of health, eroding feelings of self-worth and a sense of the meaningfulness of life. Poverty underlines other gross violations against human dignity including "social inequalities ranging from racism to gender inequality" (Singer 2009:33).

\section{Social Determinants of Health}

The three foregoing factors (coinfection, IDU and poverty) are exemplary of the syndemic extent and magnitude social determinants have on health. However, based on the HIV/AIDS research on Saskatchewan illustrated in this essay, there are two determinants of health that must be explicitly recognized: gender and ethnicity/race. Gender and ethnicity/race are paramount within the context of HIV/AIDS across Canada. In 2008, cases of HIV in Aboriginal women far exceeded all other female ethnicities, comprising 42.9 percent of all HIV positive female cases nationally, while Aboriginal men made up only 16 percent of all HIV positive male cases nationally (Public Health Agency 2010:Ch. 5). The province of Saskatchewan is especially reflective of this pattern. The HIV and AIDS in Canada Surveillance Report by the Public Health Agency of Canada (2009) stated that the proportion of women with HIV cases reported in Saskatchewan has increased steadily over the past decade. Studies also indicate that 86 percent of HIV cases reporting Aboriginal ethnicity are female, and approximately 44 percent of IDU HIV-related cases are young Aboriginal women, mostly in urban centres (Saskatchewan Ministry of Health 2010, Saskatchewan Ministry of Health 2011). Further, in 2009, data revealed that Aboriginal women accounted for a disproportionate number (93 percent) of the reported females cases of HIV under the age of thirty (Saskatchewan Ministry of Health 2012). According to Romanow, Aboriginal women with HIV/AIDS are in 'triple jeopardy' because "they are discriminated against for having HIVIAIDS and/or Hepatitis $C$, for being a minority population by virtue of their Aboriginal ancestry, and for being women" (2.4). Clearly, gender and ethnicity/race increasingly play significant roles when it comes to HIVIAIDS in urban Saskatchewan. 


\section{Structural Violence: The Boom}

\section{Province}

Skinner identified coinfection, IDU, poverty, and other social determinants of health as the primary factors accelerating the progression of HIV to AIDS in Saskatoon. More importantly, however, Dr. Skinner's work can reveal the main contributors to the HIV syndemic in Saskatoon. The emerging HIV/AIDS epidemic in Saskatchewan, and particularly in Saskatoon, illustrates how various sociocultural and health conditions are contesting with and being compromised by a multitude of political and economic factors fostering risk environments. These risk environments are primarily the result of abysmal legislation and political neglect; however, this reality is all too often subjugated by a privileged "blame the victim" attitude. Structural violence refers specifically to the social suffering and relations of inequality that are imposed upon "the poor and working classes, people of color, women and sexual minorities" (Singer 2009:33). It systemically and systematically situates marginalized persons into circumstances to keep them subordinate and struggling in order to maintain the social status quo. Indeed, "Health Canada's establishment of a hierarchy of 'high-risk' groups for contracting HIV/AIDS echoes the stratification and social inequality in the general population" (Romanow 2003:2.4).

Within the field of anthropology, structural violence is a complex and nuanced term. It acknowledges that colonialism is both an historical and an ongoing phenomenon that exerts a myriad of oppressive and repressive forces on marginalized individuals. These forces directly impact the mental and physical health of historically oppressed persons that extends into the present. The data conveyed in this paper explicitly identify the reoccurring theme of Aboriginal people, especially women, being afflicted with the worst health in urban Saskatchewan as a direct result of structural violence. The prevalence of HIVIAIDS among Aboriginal persons in urban centres such as Saskatoon is well recognized as a neocolonial phenomenon (Waldram, Herring \& Young 2006:295), and indicates that health inequality is inextricably linked to political context.

Saskatchewan has shifted from a "have-not-" to a "have-"province since the mid-199os (Globe and Mail). Paradoxically, in times of economic 'boom,' Saskatchewan's Aboriginal population continues to endure poorer health and live in low socioeconomic conditions in a class of poverty. Meili (2012:27-28) connects this social discrepancy in Saskatoon to a lack of developmental planning and Saskatchewan's notorious 'boom-bust' cycles. However, this paper asserts that correlation between poverty among Aboriginal peoples and the consequential increased risk of contracting HIVIAIDS is directly associated with political neglect, structural violence, and institutionalized racism. It is evident in the racialized neighbourhoods in inner-city Saskatoon, whose residents are susceptible not only to jeopardized health, but also family violence and/or sexual abuse, poor education, homelessness, drug and alcohol addictions as well as social stigma, racism, and sexism. Taking into account the local historical circumstances with a syndemic lens, one can recognize the harmful conditions interacting among Saskatchewan's Aboriginal populations as a result of historical and ongoing colonialism.

\section{Conclusion}

The fact that Aboriginal communities in urban Saskatchewan have been systemically oppressed through structural violence places them at substantial risk of HIV infection. The emerging HIV/AIDS syndemic in Saskatoon is powerfully correlated with co-infection, IDU, poverty, and other social determinants of health, namely gender and race/ethnicity. Clearly, there are syndemic interactions between HIV and the aforementioned factors, as well as syndemic interactions among the factors themselves. These interactions subsequently exacerbate the conditions of Aboriginal persons living in poverty and particularly those who are HIV positive. The gravity of this situation exposes a lack of political responsibility to Canada's Aboriginal peoples and inadequate public-policy decision-making. Structural violence perpetuates seemingly expired social inequalities in developed nations such as Canada. While the social determinants of health among Aboriginal persons paints a dismal picture, Singer proposes a shift in policymaking to a grassroots level - to those who bear the painful burdens of addiction, stigma, and socially-determined poverty (2009:299). It is significant to acknowledge that medical anthropologists should be critical of the social structure and "risk environments" that are pervasive among those suffering from poor health. Syndemic theory provides a gateway for medical anthropologists to hold institutions and governments accountable while guiding researchers to work against structural violence that persists over time, as seen in Saskatchewan's HIVIAIDS epidemic.

\section{References Cited}

Adam, Betty Ann

2011 Sask. Health Official says HIV-Infected Teens on Rise. Star Phoenix, December 2.

Campbell, David

2008 Learning from the Saskatchewan Miracle. Globe and Mail, July 6 (Retrieved November 26, 2012). 
DeBruyn, Theodore

1998 HIV/AIDS and discrimination: A Discussion Paper.

Montreal: Canadian HIV/AIDS Legal Network.

Downe, Pamela

2012 ANTH 332 Lecture, University of Saskatchewan.

Hammond, Karsten, Michelle Persaud, Oscar E. Larios,

Karen McClean, Athena McConnell, Stephen Sanche,

Satchan Takaya, Ben Tan, Kurt Williams and Stuart Skinner 2011 Increasing Burden of HIV/AIDS on Hospitals in

Saskatoon, Saskatchewan, Canada. Poster abstract session at the annual meeting of Infectious Diseases

Society of America, Boston, MA October 20-23.

Hays, J

2000 The Burdens of Disease: Epidemics and Human

Response in Western History. New Brunswick, NJ:

Rutgers University Press.

Lemstra, Mark, Cory Neudorf, Johnmark Opondo 2006 Health Disparity by Neighbourhood Income.

Canadian Journal of Public Health 97(6):435-439.

Meili, Ryan

2012 A Healthy Society: How a Focus on Health Can

Revive Canadian Democracy. Saskatoon: Purich

Publishing Limited.

Nichter, Mark

2008 Global Health: Why Cultural Perceptions, Social

Representations, and Biopolitics Matter. Tuscon:

The University of Arizona Press.

Peters, Evelyn J., Tyler A McCreary

2008 Poor Neighbourhoods and the Changing

Geography of Food Retailing in Saskatoon,

Saskatchewan, 1984-2004. Canadian Journal of

Urban Research 17(1):78-106.

Public Health Agency of Canada

2010 HIV/AIDS Epi Updates. Saskatchewan: Ministry of

Health.Rhodes, Tim, Merrill Singer, Phillippe

Bourgois, Samuel R. Friedman, Steffanie A.

Strathdee

2005 The Social Structural Production of HIV Risk

Among Injecting Drug Uses. Social Science \&

Medicine 61:1026-1044.

Romanow, Carol-Anne

2003 HIV/AIDS and Aboriginal Women in

Saskatchewan: Colonization, Marginalization and

Recovery. Master's Thesis, University of

Saskatchewan.

Saskatchewan Ministry of Health

2011 Saskatchewan HIV Strategy 2010-2014.

Saskatchewan Ministry of Health: Population Health

Branch

2011 HIV and AIDS in Saskatchewan 2010.

Singer, Merrill

1995 Beyond the Ivory Tower: Critical Praxis in Medical

Anthropology. Medical Anthropology Quarterly

9(1):80-106.
Singer, Merrill, Scott Clair

2003 Syndemics and Public Health: Reconceptualizing

Disease in Bio-Social Context. Medical

Anthropology Quarterly 17(4):423-441.

Singer, Merrill

2009 Introduction to Syndemics: A Critical Systems

Approach to Public and Community Health. San

Francisco: Jossey-Bass.

Singer, Merrill and Pamela I. Erickson

2011 A Companion to Medical Anthropology. United

Kingdom:Blackwell Publishing Ltd.

Skinner, Stuart

2011 Sask HIV Update. AIDS Saskatoon.

Waldram, James B., D. Ann Herring, and T. Kue Young

2006 Aboriginal Health in Canada: Historical, Cultural and Epidemiological Perspectives. Toronto:

University of Toronto Press.

University of Saskatchwan Undergraduate Research Journal 